aber ein geringeres Gewicht. Und nmgekehrt, soll jeder Winkel das bestimmte Gewicht haben, so sind schon bei zwei Gruppen, von mchreren ganz abgesehen, mehr als 2 p Einstellungen für jede Richtung erforderlich. Hiernach würde der ersten Methode der Vorzug gegeben werden müssen.
Die zweite Methode liefert unter allen Umständen geringere Resultate. Soll der summirte Winkel die gewïnschte Genauigkeit haben, so sind bei 6 Richtungen 3 p Einstellungen jeder Richtung erforderlich, bei 7... $\frac{24}{7}$ p u. s. w., sie kann daher nicht in Betracht kommen.

Bremiker.

\title{
Ueber die Bahn der Urda (167).
}

Von diesem am 28. August als 12. Grösse aufgefundenen Planeten gelangen mir die folgenden Beobachtungen, jede aus 12 Ringmikrometervergleichungen bestehend.

\begin{tabular}{|c|c|c|c|c|c|c|c|c|}
\hline \multicolumn{6}{|c|}{$(167)-*$} & \multicolumn{2}{|c|}{$\lg \left(p^{\prime \prime} \triangle\right)$} & \\
\hline 1876 & H. C. m. Zt. & $\triangle \alpha$ & $\triangle \delta$ & $\alpha$ & $\delta$ & in $\alpha$ & in $\delta$ & Vgl.-St. \\
\hline ng. 28 & $21^{\mathrm{s}}$ & $48: 47$ & $+\quad 18^{\prime \prime} 7$ & $21^{\mathrm{h}} 58^{\mathrm{m}} 32^{\mathrm{s}} 22$ & $-11^{\circ} 23^{\prime} 41^{\prime \prime} 2$ & $0.6 j 6$ & 0.839 & a \\
\hline 30 & $\begin{array}{lll}13 & 35 & 16\end{array}$ & $-1^{\mathrm{m}} 12.43$ & -34.4 & $\begin{array}{lll}21 & 57 & 2.27\end{array}$ & -113323.3 & 0.576 & 0.847 & b \\
\hline 12 & 43 & 8.37 & +150.6 & $148 \quad 16.70$ & $-1231 \quad 14$ & 0.612 & 0.848 & c \\
\hline 1 & $12 \quad 19 \quad 28$ & $\begin{array}{l}27.84 \\
+\quad 2\end{array}$ & 3.0 & $21 \quad 46 \quad 38.63$ & $\begin{array}{lll}-12 & 42 & 24.8\end{array}$ & 0.571 & 0.852 & d \\
\hline
\end{tabular}

Hierbei sind die Vergleichsterne für 1876.0 folgendermaassen angenommen:
a $21^{\mathrm{h}} 59^{\mathrm{m}} 16^{\mathrm{s}} 94-11^{0} 24^{\prime} 14^{\prime \prime} 8$
10. Gr.; best. dur.h 3
Fad.-Mikr.-Vergl. mit
$22 \quad 3 \quad 54.90$
2546.95 (W. $22 \mathrm{~h} 34)$
Cat. 1850.
b $\quad 21 \quad 58 \quad 10.94-1133 \quad 3.8 \quad 10.5$ Gr.; best. durch 5 Fad.-Mikr.-Vergl. mit $\begin{array}{lllll}58 & 3.56 & 28 & 55.1 & \text { W. } 21^{\mathrm{h}} 1314 .\end{array}$
c $\quad 2148 \quad 21.29-1233 \quad 18.7 \quad$ Mittel aus: $21.29 \quad 21.8 \quad$ W. $21^{\text {h }} 1106$ (Gew. 1) 21.2518 .2 Lamont 3783 (Gew. 4) $21.31 \quad 18.4$ Y. 9600 (Gew. 5 und 3) d $2146 \quad 7.01-124241.6 \quad 10.5$ Gr.; best. durch 3
$41 \quad 22.87$
23.11
22.63
43 4.5 Mittel aus:
4.1 W. $21^{\text {b966 }}$ und
4.9 Lam. 3761.

Es scheint nicht, dass der Planet sonst noch beobachtet worden. Aus diesem dürftigen Material ist also die Babn herzuleiten. Zu dem Ende wurde zuerst durch die beiden äussersten Orte eine Kreisbahn gelegt, welche diente, die Beobachtungen von Aberration und Parallaxe zu befreien. Wie die spätere Rechnung zeigte, waren so diese Reductionen hinreichend genau erhalten worden. Nachdem dann die Beobachtungen auf das gemeinschaftliche Aequinoctium von 1876.0 reducirt und in Längen und Breiten verwandelt, und die Sonnenorte zur Wegschaffung der Sonnenbreite auf den locus fictus bezogen waren, wurden die 4 Längen und die beiden äussersten Breiten benutzt zur Bahnbestimmıng. Selbst in diesem recht ungïnstigen Falle fiihrte die
Anordnung, welche Oppolzer den successiven Annäherungen gegeben hat, nach wenigen $V$ ersuchen zum Ziele. Auch ergab sich die Bahn mit weit geringerer Unbestimmtheit, als ich erwartet hatte. Die resultirenden Elemente sind die folgenden:

Epoche: 1876. Jan. 0.0 m. Zt. Berlin

$$
\begin{aligned}
& \mathrm{L}=317^{\circ} 43^{\prime} 27^{\prime \prime} 4 \\
& \pi=323922.2+50^{\prime \prime} 24 . \mathrm{t} \\
& \delta=170725.4+49.25 . \mathrm{t} \\
& \mathrm{i}=14214.5-0.47 . \mathrm{t} \\
& \boldsymbol{\varphi}=181030.6 \\
& \mu=614^{\prime \prime} 475 \\
& \log \mathrm{a}=0.507668
\end{aligned}
$$

$t$ in Jahren von der Epoclıe an gezählt.

Durch Wiederberechnung der Beobachtungen hieraus erhïlt man die Fehler $(\mathrm{C}-\mathrm{O})$ :

$$
\begin{array}{ccccrr} 
& & \text { Länge } & \text { Breite } & \text { AR. } & \text { Decl. } \\
\text { Aug. } & 28 & 0^{\prime \prime} 0 & 0^{\prime \prime} 0 & 0^{\circ} 00 & 0^{\prime \prime} 0 \\
\text { n } & 30 & 0.0 & +1.1 & -0.02 & +1.2 \\
\text { Sept. } & 12 & +0.1 & +-4.9 & -0.10 & +4.7 \\
\text { n } & 15 & 0.0 & 0.0 & 0.00 & 0.0
\end{array}
$$

Es ist zu bedauern, dass keine weiteren Beobachtungen dieses Planeten erlangt worden sind, da die Elemente etwas sehr merkwürdiges zeigen. Hält man sie nämlich mit denen von Gerda (122) zusammen, welche, auf dieselbe Epoche 1876.0 reducirt, auf Minuten abgekürzt, sind (Berl. Jahrb. 1878) :

$$
\begin{aligned}
& \mathrm{L}=168^{\circ} 46^{\circ} \\
& \pi=20853 \\
& \Omega=179 \quad 1 \\
& \mathrm{i}=136 \\
& \varphi=29
\end{aligned}
$$




$$
\begin{aligned}
& \mu=614^{\prime \prime} 1 \\
& \log a=0.50784
\end{aligned}
$$

so findet man 3 der Elemente, nämlich Knoten, Neigung und halbe grosse Axe, fast genau gleich. Die Excentricität ist zwar bei Urda bedeutend grösser erhalten worden; aber der gefundene Werth kann natürlich ganz bedeutende Aenderungen erleideu, da er auf einem so kleinen Bogenstïck (von 18 Tagen Intervall) beruht. Aus demselben Grunde bleibt auch die Richtung znm Perihel einer grossen Unsicherheit unterworfen. Es scheint demnach, dass in Urda's Bahn möglicherweise sogar 5 Elemente mit denen Grerda's identisch sein könnten. Das 6. Element, die Länge in der Bahn, negirt nun aber entschieden die Identität der Planeten selbst. Und aller Zweifel wird dadurch gehoben, dass Gerda in der Opposition im vorangehenden April auf der Berliner Sternwarte mehrere Mal wirklich beobachtet worden ist, nahe dem von Herrn Stockwell's Rechnungen angegebenen Ort.

Der Nachweis zweier in derselben Ebene und, so viel man bis jetzt annehmen muss, mit derselben Umlaufszeit die Sonne umkreisenden Planetoiden hat ein grosses Interesse, und es ist zu hoffen, dass Urda in der nächsten Opposition, die in Januar 1878 stattfindet, und wo der Planet die Grösse 12.7 haben wird, wieder aufgefunden werde.

Hamilton College, 5. Jan. 1877.

\section{Schreiben des Herrn Dr. Seeliger an den Herausgeber.}

In meiner kürzlich erschienenen Abhandlung über die Theorie des Heliometers sind einige Druckfelıler, trotz der Sorgfalt, die ich auf die Correctur verwendet, steben geblieben. Da einige von ihnen zu Missverständnissen Anlass reben könnten, möchte ich Sie bitten, die folgenden Verbesserungen in den Astron. Nachr. zu erwälınen. Dieselben sind mir zum grossen Theil von Herrn Prof. Brulıns und Herrn stud, astr. Har\%er mitgetheilt worden. Einige im Folgenden nicht erwähnte Druckfehler gebe ich deshalb nicht an, weil dieselben ohne Weiteres beim Lesen in die Augen fallen.
S. 10 in Formel (3) $+\mathrm{y} \operatorname{tg} \delta \cos t$ statt $-y \operatorname{tg} \delta \cos t$

, 19, Zeile 9 v. u. $v-\pi-t=180^{\circ}$ statt $v-\pi-t=0^{\circ}$

\#27,, 9 , o. $\mathrm{m} \cos \lambda$ statt $\mu \cos \lambda$

"62,, 10 ,, fehlt auf der rechten Seite der Factor $\pi$

»86,, 3 , u. Poldistanz statt Declination

, 88 in den 4 ersten Formeln vertausche $M$ in $N$

88, Leile 7 v. u. $\mathrm{C}=-u \cos \Theta \cos \varepsilon$ statt $\mathrm{C}=-\varkappa \cos \Theta$

" 89,, 2 , o. lies $\cos \Theta \cos \varepsilon$ fïr $\cos \Theta \sin \varepsilon$

$\eta 89, \quad 3 n, \quad n \cos \Theta \sin \varepsilon \quad \eta \sin \Theta \sin \varepsilon$

$\eta 89, \Rightarrow 8 \%, \Rightarrow \sin P$ für $\cos P$

» 96 , es ist hier überall $M$ für $m$ zu setzen.

Bonn, den 11. Januar 1877.

\section{The New Star in the Swan.}

On January 9th the sky was unusually clear and the Spectrum of Dr. Schmidt's Nova came out with amazing sharpness and brilliancy. In addition to the 5 bright lines seen on the $2^{\text {nd }}$, two others were detected: viz

\section{Mill.mm}

N.. 1a W. L. 594 Very narrow line

$» 7 \gg n 414 \pm$ Excessively faint, but still certainly and repeatedly seen.

Between wave-lenghts 655 and 594 the Spectrum was certainly banded, and, most probably, there were two additional faint maxima of brilliancy in that interval. The continuous spectrum attains a maximum in the region about W. L. 525 and extends, though possibly not witout interruption, as far as the faint line Nr. 7 . The star was estimated of $7^{\text {th }}$ magnitude and was of a red colour with a decided tinge of purple, reminding me forcibly of the varieties of red produced by the quarz-plate in Zöllner's photometer.

Lord Lindsay's Observatory Dunecht,

$$
\text { 13. Jan. 1877. Ralph Copeland. }
$$

I n h a l t:

Zu N. 2117. Bremiker. Leher Winkelmessung und Ansgleichung. 65. - C. H. F. Peters. Ucber die Bahn der Urda (167). 77. H. Seeliger. Schreiben an den Herausgeber. 79. - Ralph Copeland. The New Slar in the Swan. 79.

Kiel. 1877, Februar 10. - Druck von Fiencke \& Schachel in Kiel. 